\title{
Opportunistic Cooperative Diversity with Feedback and Cheap Radios
}

\author{
Aggelos Bletsas, Member, IEEE, Ashish Khisti, Student Member, IEEE, and Moe Z. Win, Fellow, IEEE
}

\begin{abstract}
Practical cooperative diversity protocols often rely on low-cost radios that treat multiple in-band signals as noise and thus require strictly orthogonal transmissions. We analyze the performance of a class of opportunistic relaying protocols that employ simple packet level feedback and strictly orthogonal transmissions. It is shown that the diversity-multiplexing tradeoff of the proposed protocols either matches or outperforms the multi-input-single-output (MISO), zero-feedback performance. These gains indicate that low complexity radios and feedback could be an appealing architecture for future user cooperation protocols.
\end{abstract}

Index Terms - Network cooperative diversity, outage probability, fading channel, virtual antenna arrays, cheap radios, wireless networks.

\section{INTRODUCTION}

$\mathbf{C}$ OOPERATIVE transmissions from distributed terminals continue to attract considerable interest especially in studies of the quasi-static (non-ergodic) relay channel. On the theoretical side, information theoretic analysis of several user cooperation protocols is presented in [1]-[6]. These works focus on fundamental limits of cooperative transmission and assume idealistic conditions such as perfect synchronization across the relays, simultaneous in-band transmissions (both frequency and time) and availability of practical codes that approach the random coding performance. For example, the protocols in [1] require simultaneous in-band transmissions among a set of decode-and-forward (DF) relays that form a distributed antenna and utilize optimal space-time coding (STC). Subsequent research has shown that if the principle of both time and frequency in-band transmissions (PTFIT) is further exploited, end-to-end performance can be enhanced. For example, in [2] it has been shown that when the source continues to transmit and a single DF or amplify-and-forward (AF) relay re-transmits, channel degrees-of-freedom are not wasted, improving performance compared to schemes where

Manuscript received February 15, 2007; revised April 30, 2007; accepted July 23, 2007. The associate editor coordinating the review of this paper and approving it for publication was H. Jafarkhani. This research was supported in part by the National Science Foundation under Grants ANI-0335256 and ECS-0636519. Parts of this work were presented in ACM International Conference on Wireless Communications and Mobile Computing, July 2006, Vancouver Canada and MSRI Workshop on Mathematics of Relaying, April 2006, Berkeley California.

A. Bletsas was with the Media Laboratory, Massachusetts Institute of Technology, 77 Massachusetts Avenue, Cambridge, MA 02139 USA. He is now with Radiocommunications Laboratory (RCL), Department of Physics, Aristotle University of Thessaloniki, Greece 54124 (e-mail: bletsas@auth.gr).

A. Khisti is with the Research Laboratory of Electronics (RLE), Massachusetts Institute of Technology, Bldg 36-683, 77 Massachusetts Avenue, Cambridge, MA 02139 USA (e-mail: khisti@mit.edu).

M. Win is with the Laboratory for Information and Decision Systems (LIDS), Massachusetts Institute of Technology, 77 Massachusetts Avenue, Cambridge, MA 02139 USA (e-mail: moewin@mit.edu).

Digital Object Identifier 10.1109/TWC.2008.070193. source stops transmitting during relay retransmission [3]. Similar conclusions appear in [4]-[6].

On the practical side, there has been recent interest in approaching the random coding performance limits with distributed space time codes (see e.g., [7] and references therein), under the assumption of PTFIT. We note however that the PTFIT is not supported by many existing low-cost radios, which treat multiple in-band signals as noise. One common reason is that many existing RF-front ends are not linear and thus, the superposition of signals at the receiver antenna does not appear as a linear combination at baseband [8]. Consecutively, cooperative diversity techniques based on the PTFIT, are not applicable with many existing, low-cost radios. In an effort to utilize low-cost radios in cooperative settings that adhere to principles of orthogonal transmissions (POT), the research community has proposed single transmission of a selected relay among a set of possible candidates, orthogonally (not in-band) to the source. For example, in [9] the relay geometrically closer to destination forwards information, among the DF relays that have successfully decoded the information from the original source. All network terminals were assumed with GPS receivers and distributed selection was envisioned alongside the lines of geographic forwarding [10]. MonteCarlo simulations provided performance results in Rayleigh Fading, incorporating feedback from destination.

Adhering to the principle of orthogonal transmissions (POT), a relay selection scheme among low-cost DF or AF relays, based on pro-active channel measurements and forwarding orthogonally to the source, was proposed in [11]. A simple protocol was analyzed, allowing fast relay selection with limited delay (within a fraction of the channel coherence time), in a distributed manner. Diversity-multiplexing tradeoff (DMT) analysis of no-feedback setups in Rayleigh Fading revealed no performance loss, compared to distributed spacetime coding schemes that adhere to PTFIT. That result demonstrated that the selection diversity benefits found in the classic multi-antenna (MIMO) literature (e.g. [12], [13]) carry over in the relay channel, even though the latter is fundamentally different than the classic MIMO channel: information is not apriori known at the relays as opposed to the co-located multiantenna case, but instead, messages need to be conveyed over distributed and noisy links between source and receiving relay antennas.

Treating low-complexity relay terminals as distributed sensors of the wireless channel (and not necessarily as active retransmitters) is the main theme of opportunistic relaying [14]. Experimental testing of opportunistic cooperative diversity in existing radios can be found in [8] and [14]. Subsequent finite-SNR analysis revealed the advantages of opportunistic relaying over certain space-time coding schemes with DF [15] 
or AF relays [16].

In this letter, we study the performance of opportunistic cooperative diversity in the high spectral efficiency regime via packet level feedback. While we adhere to strictly orthogonal transmissions facilitated by existing, low-cost (cheap) radios, we analyze the performance of Gaussian codebooks. To put our work in perspective,

1) in contrast to [11], where no-feedback schemes under Rayleigh fading are analyzed, we study feedback with opportunistic relaying under generalized fading, where Rayleigh is a special case. Moreover, we provide DMT analysis for more than one relay selection policies;

2) in contrast to [3], where a single DF relay with feedback under Rayleigh fading is studied, we analyze a multiple AF relay setup under generalized fading;

3) in contrast to [17], where feedback with DF relays transmitting in-band with the source is analyzed, we study feedback with multiple AF relays transmitting orthogonally to the source and each other (POT);

We eventually find out, that even under the POT, performance is significantly improved in the high spectral efficiency regime, compared to the no-feedback case, allowing direct exploitation of cooperative diversity benefits with cheap and existing radios.

In section II we describe the basic notation, system assumptions and definitions followed throughout this document, in section III we quantify the performance as a function of feedback rounds and finally, in section IV we discuss the findings.

\section{System Model And Definitions}

The setup includes one source node, one destination node, and a set $\mathcal{S}_{\text {relay }}=\{1,2, \ldots, K\}$ of $K$ amplify-and-forward (AF) relay nodes. We consider a conventional quasi-static (slow), flat fading model (see e.g. [1], [3], [4]), where the received signal in a link $(\mathrm{A} \rightarrow \mathrm{B})$ between two nodes " $\mathrm{A}$ " and " $\mathrm{B}$ " is given by:

$$
y_{\mathrm{B}}[j]=a_{\mathrm{AB}} \cdot x_{\mathrm{A}}[j]+n_{\mathrm{B}}[j]
$$

where $x_{\mathrm{A}}[j]$ is the $j$-th symbol transmitted from node $\mathrm{A}$ and $n_{\mathrm{B}} \sim \mathcal{C} \mathcal{N}\left(0, N_{0}\right)$ is the additive white Gaussian noise (AWGN) at node $\mathrm{B}^{1}{ }^{1}$ The complex valued channel gain, $a_{\mathrm{AB}}$, between the link $\mathrm{A} \rightarrow \mathrm{B}$, is distributed according to a Nakagami- $m$ distribution, ${ }^{2}$ i.e., $\left|a_{\mathrm{AB}}\right|^{2}$ follows a gamma distribution with shape $m_{\mathrm{AB}} \equiv m>0$ and scale $\theta_{\mathrm{AB}} \equiv \theta>0$, with pdf given by:

$$
f_{\left|a_{\mathrm{AB}}\right|^{2}}(x)=x^{m-1} \frac{\exp (-x / \theta)}{\Gamma(m) \theta^{m}},
$$

where $\Gamma(\cdot)$ is the complete gamma function (see (26) in appendix). We denote the transmit SNR as $\rho=\mathbb{E}\left\{\left|x_{\mathrm{A}}\right|^{2}\right\} / N_{0}$.

We utilize the diversity-multiplexing tradeoff (DMT) to evaluate performance. This framework was introduced by

\footnotetext{
${ }^{1} \mathcal{C N}\left(\mu, \sigma^{2}\right)$ denotes a complex circularly symmetric Gaussian distribution with mean $\mu$ and variance $\sigma^{2}$.

${ }^{2}$ Nakagami- $m$ encompasses a variety of fading models followed in the literature. For example, $m=1$ corresponds to Rayleigh fading, while $m=$ $\frac{(\kappa+1)^{2}}{2 \kappa+1}>1$ approximates Ricean fading ( $\kappa$ is the Ricean factor).
}

Zheng and Tse [18] for fixed rate codes and subsequently extended to variable rate codes in [19].

Definition 1 (Diversity-Multiplexing Tradeoff [18], [19]): Consider a sequence of variable rate codes $C_{\rho}$, indexed by SNR $(\rho)$. If $P_{e}(\rho)$ and $R_{e}(\rho)$ denote the outage probability and the average rate (bits per channel use) associated with $C_{\rho}$, then the multiplexing gain $r_{e}$ and diversity order $d$ are defined as:

$$
r_{e} \triangleq \lim _{\rho \rightarrow \infty} \frac{R_{e}(\rho)}{\log \rho}, \quad d \triangleq-\lim _{\rho \rightarrow \infty} \frac{\log P_{e}(\rho)}{\log \rho} .
$$

In the sequel, operators $\doteq, \dot{\leq}$ are used to simplify presentation:

Definition 2: A function $f(\rho)$ is said to be exponentially equal to $b$, denoted by $f(\rho) \doteq \rho^{b}$, if

$$
\lim _{\rho \rightarrow \infty} \frac{\log f(\rho)}{\log \rho}=b .
$$

Relation $\dot{\leq}$ is defined in a similar fashion.

\section{A. Opportunistic Relay Selection}

Whenever a feedback signal from the final destination flags the necessity of a relay transmission, a specific relay in the set $\mathcal{S}_{\text {relay }}$ is selected according to the following criteria:

$$
\mathrm{b}=\arg \max _{k \in \mathcal{S}_{\text {relay }}}\left\{g\left(\left|a_{\mathrm{S} k}\right|^{2},\left|a_{k \mathrm{D}}\right|^{2}\right)\right\},
$$

with two choices (policies) for function $g(\cdot, \cdot)$ :

$$
\begin{aligned}
\text { policy I (min): } & g(x, y)=\min \{x, y\},(6) \\
\text { policy II (harmonic mean): } & g(x, y)=\frac{2 x y}{x+y} .
\end{aligned}
$$

Such opportunistic relay selection is proposed in [11] and [14], where its practical and theoretical properties are discussed. In particular, opportunistic relay selection can be performed via distributed techniques that do not require global channel state information (CSI) at a central controller or anywhere else in the network and incurs a small fraction (e.g. two orders of magnitude) of the channel coherence time. The interested reader could refer to [11], [14] for additional details regarding opportunistic relay selection algorithms, their associated overhead and their implementation in actual networks.

\section{Opportunistic AF Relaying With FeEdBACK}

\section{A. Single Round of Feedback}

The transmission of each message requires $n$ channel uses. The source transmits with $n$ channel uses and the signals received at the best relay and the destination are given by:

$$
\begin{aligned}
y_{\mathrm{b}}[j] & =a_{\mathrm{Sb}} \cdot x[j]+n_{\mathrm{b}}[j], \quad j=1,2 \ldots n, \\
y_{\mathrm{D}}[j] & =a_{\mathrm{SD}} \cdot x[j]+n_{\mathrm{D}}[j] .
\end{aligned}
$$

In our analysis, we consider a sequence of Gaussian codebooks $C_{\rho}$ with nominal rate

$$
R(\rho)=\log \left(1+\rho^{r}\right)
$$


for some $r \in(0,1)$. Accordingly, the decoding at the destination fails if the following event happens:

$$
\begin{aligned}
\mathcal{E}_{1} & \triangleq\left\{\mathcal{I}_{0}=n \log \left(1+\rho\left|a_{\mathrm{SD}}\right|^{2}\right) \leq n R(\rho)\right\} \\
& =\left\{\left|a_{\mathrm{SD}}\right|^{2} \leq \rho^{r-1}\right\} .
\end{aligned}
$$

If the selected relay $b$ receives a single-bit feedback from the destination, indicating that the message has not been decoded correctly, it then transmits $x_{b}[i]=\beta y_{d}[i]$ over the next $n$ slots, where $\beta$ is a properly normalized amplification factor, as in [3]. The received symbols at the destination are given by:

$$
z_{\mathrm{D}}[i]=a_{\mathrm{bD}} \cdot x_{\mathrm{b}}[i]+n_{\mathrm{D}}[i], \quad i=1,2, \ldots, n .
$$

With $\mathrm{AaF}$ relays, the end-to-end mutual information is given by [3]:

$$
\mathcal{I}_{\mathrm{AF}}^{F}=n \log \left(1+\rho\left|a_{\mathrm{SD}}\right|^{2}+f\left(\rho\left|a_{\mathrm{bD}}\right|^{2}, \rho\left|a_{\mathrm{Sb}}\right|^{2}\right)\right),
$$

where $f(x, y)=\frac{x y}{x+y+1}$. The single relay re-transmission will fail if

$$
\begin{aligned}
\mathcal{E}_{2} & \triangleq\left\{\mathcal{I}_{\mathrm{AF}}^{F} \leq n R(\rho)\right\} \\
& =\left\{\left|a_{\mathrm{SD}}\right|^{2}+\frac{1}{\rho} f\left(\rho\left|a_{\mathrm{bD}}\right|^{2}, \rho\left|a_{\mathrm{Sb}}\right|^{2}\right) \leq \rho^{r-1}\right\} .
\end{aligned}
$$

Because of the feedback mechanism, the average spectral efficiency $R_{e}(\rho)$ for the code $C_{\rho}$ is given by:

$$
R_{e}(\rho)=R(\rho) \mathbb{P}\left\{\overline{\mathcal{E}_{1}}\right\}+\frac{R(\rho)}{2} \mathbb{P}\left\{\mathcal{E}_{1}\right\}
$$

where $\overline{\mathcal{E}_{1}}$ is the complementary event of $\mathcal{E}_{1}$. Thus it follows that $R_{e}(\rho) \leq R(\rho)$. Nevertheless, the effective multiplexing gain does not reduce due to feedback.

Lemma 1: The effective multiplexing gain, $r_{e}$ in Def. 1, satisfies $r_{e}=r$.

Proof: Substituting (10) in (14) we have that

$$
\begin{aligned}
R_{e}(\rho) & =R(\rho) \mathbb{P}\left\{\left|a_{\mathrm{SD}}\right|^{2} \geq \rho^{r-1}\right\}+\frac{R(\rho)}{2} \mathbb{P}\left\{\left|a_{\mathrm{SD}}\right|^{2} \leq \rho^{r-1}\right\} \\
& =R(\rho)-\frac{R(\rho)}{2} \mathbb{P}\left\{\left|a_{\mathrm{SD}}\right|^{2} \leq \rho^{r-1}\right\} \\
& \doteq R(\rho)-\frac{R(\rho)}{2} \rho^{(r-1) m_{\mathrm{SD}}} \\
& \doteq R(\rho) .
\end{aligned}
$$

where we have used that $\mathbb{P}\left\{\left|a_{\mathrm{SD}}\right|^{2} \leq \rho^{r-1}\right\} \doteq \rho^{(r-1) m_{\mathrm{SD}}}$ according to the Lemma 2 in the appendix.

Thus we have that

$$
r_{e}=\lim _{\rho \rightarrow \infty} \frac{R_{e}(\rho)}{\log \rho}=\lim _{\rho \rightarrow \infty} \frac{R(\rho)}{\log \rho}=r,
$$

as required.

Next we compute the outage probability $P_{e}(\rho)$ in Def. 1 .

$$
\begin{aligned}
& P_{e}(\rho)=\mathbb{P}\left\{\mathcal{E}_{1} \bigcap\left(\mathcal{E}_{2} \mid \mathcal{E}_{1}\right)\right\}=\mathbb{P}\left\{\mathcal{E}_{1} \bigcap \mathcal{E}_{2}\right\} \\
& =\mathbb{P}\left\{\left|a_{\mathrm{SD}}\right|^{2} \leq \rho^{r-1}, \quad\left|a_{\mathrm{SD}}\right|^{2}+\frac{1}{\rho} f\left(\left|a_{\mathrm{Sb}}\right|^{2} \rho,\left|a_{\mathrm{bD}}\right|^{2} \rho\right)<\rho^{r-1}\right\} \\
& \leq \mathbb{P}\left\{\left|a_{\mathrm{SD}}\right|^{2} \leq \rho^{r-1}, f\left(\left|a_{\mathrm{Sb}}\right|^{2} \rho,\left|a_{\mathrm{bD}}\right|^{2} \rho\right)<\rho^{r}\right\} \\
& =\mathbb{P}\left\{\left|a_{\mathrm{SD}}\right|^{2} \leq \rho^{r-1}\right) \times \mathbb{P}\left(f\left(\left|a_{\mathrm{Sb}}\right|^{2} \rho,\left|a_{\mathrm{bD}}\right|^{2} \rho\right)<\rho^{r}\right\}
\end{aligned}
$$

where (16) follows by substituting (10) and (13) in (15) and we use the fact that for any positive $\theta$,

$$
\mathbb{P}(X+Y<\theta) \leq \mathbb{P}(X \leq \theta, Y \leq \theta),
$$

for any positive random variables $X$ and $Y$ in (17), and (18) follows from the fact that the random variables $\left|a_{\mathrm{SD}}\right|^{2}$ and $\left(\left|a_{\mathrm{Sb}}\right|^{2},\left|a_{\mathrm{bD}}\right|^{2}\right)$ are mutually independent.

We separately upper bound the two terms in (18). First from Lemma 2 in appendix, we have that with $m_{0}=m_{\mathrm{SD}}$,

$$
\mathbb{P}\left\{\left|a_{\mathrm{SD}}\right|^{2} \leq \rho^{r-1}\right\} \leq \rho^{(r-1) m_{0}}
$$

Next, using Lemma 4 in [11], we have that,

$$
\begin{aligned}
& \mathbb{P}\left\{f\left(\left|a_{\mathrm{Sb}}\right|^{2} \rho,\left|a_{\mathrm{bD}}\right|^{2} \rho\right) \leq \rho^{r}\right\} \\
\leq & \mathbb{P}\left\{\min \left\{\left|a_{\mathrm{Sb}}\right|^{2},\left|a_{\mathrm{bD}}\right|^{2}\right\} \leq \rho^{r-1}+\rho^{0.5 r-1} \sqrt{1+\rho^{r}}\right\} \\
\doteq & \mathbb{P}\left\{\min \left\{\left|a_{\mathrm{Sb}}\right|^{2},\left|a_{\mathrm{bD}}\right|^{2}\right\} \leq \rho^{r-1}\right\} \\
\doteq & \rho^{(r-1) \sum_{k=1}^{K} m_{k}}
\end{aligned}
$$

where (20) follows from Lemma 2 in the appendix with $m_{k}=$ $\min \left\{m_{\mathrm{S} k}, m_{k \mathrm{D}}\right\}$. Combining (19) and (20), we have that

$$
P_{e}(\rho) \dot{\leq} \rho^{(r-1) \sum_{k=0}^{K} m_{k}} .
$$

We summarize our analysis below.

Theorem 1: Opportunistic cooperative diversity with a single round of feedback and relay selection according to (6) or (7), achieves DMT performance $d\left(r_{e}\right)$ at least as good as $d^{*}\left(r_{e}\right)=\left(1-r_{e}\right) \sum_{k=0}^{K} m_{k}$, for $r_{e} \in(0,1)$.

Proof: From inequality (21) and the definition of diversity order in (3), we find out:

$$
\begin{aligned}
& P_{e}(\rho) \dot{\leq} \rho^{-(1-r) \sum_{k=0}^{K} m_{k}}=\rho^{-\left(1-r_{e}\right) \sum_{k=0}^{K} m_{k}} \\
\Rightarrow & d\left(r_{e}\right) \geq d^{*}\left(r_{e}\right)=\left(1-r_{e}\right) \sum_{k=0}^{K} m_{k}
\end{aligned}
$$

We note that the calculated $d^{*}\left(r_{e}\right)$ for DMT performance is a pessimistic bound, given that protocol performance is calculated as good as or better than $d^{*}\left(r_{e}\right)$. For the special case of Rayleigh fading where $m_{k}=1$,

$$
d^{*}(r)=(1-r)(K+1)
$$

corresponds to a classic multi-input single-output (MISO) antenna system without feedback, suggesting that intelligent cooperation is fruitful, even when strictly orthogonal transmissions and cheap radios are utilized. 


\section{B. Multiple Rounds of Feedback}

In certain systems with relaxed delay constraints, multiple rounds of feedback are permissible and the performance can be significantly enhanced [19]. We consider a system which allows for $L$ rounds of feedback, where a round consists of i) transmission from the source first and then, ii) subsequent transmission on a separate slot from the selected ("best") relay. An outage is declared if the destination fails to decode after $L$ rounds. Accordingly, in round 1, the source first transmits for $n$ channel uses, as in Section III-A. The destination sends a feedback message of negative acknowledgment (NACK) if it fails to decode the message from the source. The best relay then sends $n$ symbols using AF and the destination attempts to decode at the end of this transmission. This source-relay transmission constitutes a single round. If the destination fails to decode at the end of this round, then it again broadcasts a NACK to the source and the source begins transmission in round 2. This process continues until either the destination is successful in decoding or $L$ rounds are exhausted. Note that the destination transmits a total of $2 L-1$ NACK single-bit messages before a failure is declared.

In this setup we assume that the channel gains $\left|a_{\mathrm{Sb}}\right|,\left|a_{\mathrm{SD}}\right|,\left|a_{\mathrm{bD}}\right|$ remain fixed over the $L$ rounds. Assuming independent Gaussian codebooks used in each round, the total mutual information after $L$ rounds is $L$ times the mutual information in each round, given in (22). An outage occurs if the destination cannot decode after $L$ rounds:

$$
\begin{aligned}
\mathcal{E}_{3}^{L} & \triangleq\left\{\mathcal{I}_{\mathrm{AF}}^{F, L} \leq n R(\rho)\right\}, \\
\mathcal{I}_{\mathrm{AF}}^{F, L} & =n L \log \left(1+\rho\left|a_{\mathrm{SD}}\right|^{2}+f\left(\rho\left|a_{\mathrm{bD}}\right|^{2}, \rho\left|a_{\mathrm{Sb}}\right|^{2}\right)\right) .
\end{aligned}
$$

The analysis for outage probability is analogous to (15)-(20), but with $R(\rho)$ replaced by $R(\rho) / L$, and the average rate satisfies $R_{e}(\rho) \doteq R(\rho)$. It can be seen that the DMT lower bound is given by:

$$
d^{*}\left(r_{e}\right)=\left(1-\frac{r_{e}}{L}\right) \sum_{k=0}^{K} m_{k} .
$$

For the special case of Rayleigh fading, $d^{*}\left(r_{e}\right)$ becomes:

$$
d^{*}\left(r_{e}\right)=\left(1-\frac{r_{e}}{L}\right)(K+1)
$$

\section{Discussion}

The calculated DMT bounds are depicted in Fig. 1, including opportunistic relaying without feedback. The increased diversity order observed, comes from the fact that the selected relay is chosen opportunistically. For the high spectral efficiency regime $(0.5<r<1)$, strictly orthogonal transmissions from single-antenna, half-duplex radios with opportunistic cooperative diversity and single round of feedback improve on the MISO bound. ${ }^{3}$ Additional rounds of feedback further enhance performance. Spectral efficiency is significantly improved compared to the no-feedback opportunistic relaying case, simply because relay retransmission is used only when it is needed.

\footnotetext{
${ }^{3}$ multiple-input-single-output without feedback.
}

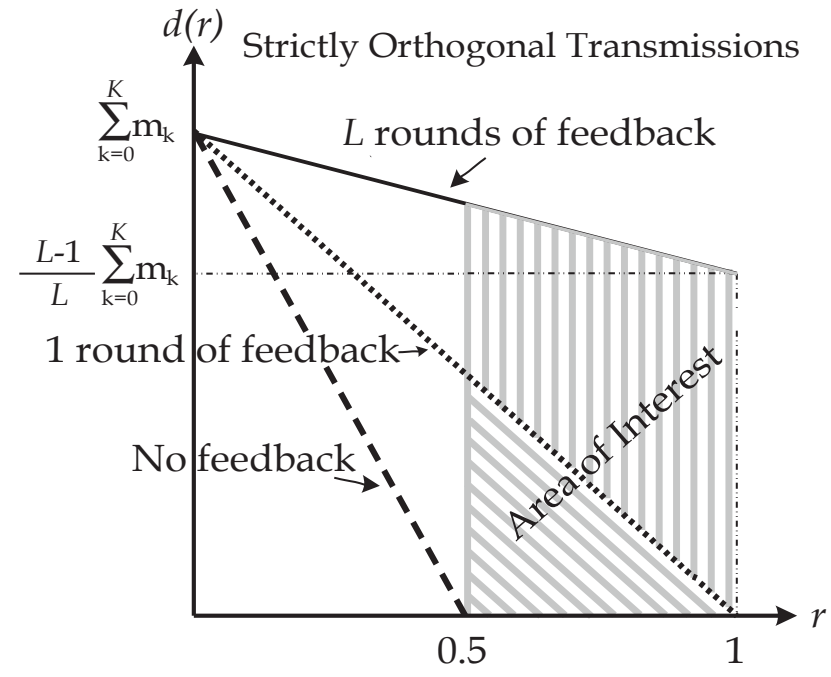

Fig. 1. Opportunistic AF relaying and one round $(L=1)$ of single-bit feedback improve on the full DMT curve, even though strictly orthogonal transmissions are used. Additional rounds $L$ of feedback with opportunistic $\mathrm{AF}$ relaying further improve the DMT performance. Note that for the special case of Rayleigh Fading, $\sum_{k=0}^{K} m_{k}=K+1$.

On the contrary, the no-feedback opportunistic protocol always uses the selected relay, which is wasteful in terms of the channel degrees-of-freedom, when the destination receives successfully the message directly from the source. The protocols proposed in [1]-[6]. provide an alternate way to efficiently utilize the channel degrees-of-freedom based on PTFIT, but their applicability to low cost radios remains to be seen.

As a final remark, we note that the NACK packet from the destination to the source needs to be transmitted when the link from the source to destination is in a deep fade. If the channel obeys reciprocity, this NACK packet may not be received by the source. In practice this problem can be alleviated by the relay node, which could retransmit the NACK packet to the source, at the cost of a small additional overhead.

\section{CONCLUSION}

Opportunistic cooperative diversity with feedback provides substantial gains at the high spectral efficiency regime, even though strictly orthogonal transmissions are utilized. In that way, existing, simple and cheap radios, built according to noncooperative principles (POT) can be employed.

\section{APPENDIX}

Lemma 2: Let $a_{\mathrm{S} k}$ and $a_{k \mathrm{D}}$ denote the channel gains from source to relay $k$ and relay $k$ to destination respectively, with $k \in \mathcal{S}_{\text {relay }}=\{1, \ldots, K\}$. The channel gains are assumed independent Nakagami- $m$ random variables (not necessarily identically distributed), with parameters $m_{\mathrm{S} k}, \theta_{\mathrm{S} k}$ and $m_{k \mathrm{D}}, \theta_{k \mathrm{D}}$ respectively. Suppose that $a_{\mathrm{Sb}}$ and $a_{\mathrm{bD}}$ denote the channel gain of the source to the selected relay and the selected relay to the destination respectively, where the selected ("best") relay is chosen according to policy I, i.e.

$$
\min \left(\left|a_{\mathrm{Sb}}\right|^{2},\left|a_{\mathrm{bD}}\right|^{2}\right)=\max _{k \in \mathcal{S}_{\text {relay }}}\left\{\min \left\{\left|a_{\mathrm{S} k}\right|^{2},\left|a_{k \mathrm{D}}\right|^{2}\right\}\right\},
$$


or is chosen according to policy II, i.e.

$$
\frac{\left|a_{\mathrm{Sb}}\right|^{2}\left|a_{\mathrm{bD}}\right|^{2}}{\left|a_{\mathrm{Sb}}\right|^{2}+\left|a_{\mathrm{bD}}\right|^{2}}=\max _{k \in \mathcal{S}_{\text {relay }}}\left\{\frac{\left|a_{\mathrm{S} k}\right|^{2}\left|a_{k \mathrm{D}}\right|^{2}}{\left|a_{\mathrm{S} k}\right|^{2}+\left|a_{k \mathrm{D}}\right|^{2}}\right\} \text {. }
$$

Then, for any $v>0$, the following relations hold:

1) $\mathbb{P}\left(\min \left\{\left|a_{\mathrm{Sb}}\right|^{2},\left|a_{\mathrm{bD}}\right|^{2}\right\} \leq \rho^{-v}\right) \doteq \rho^{-v \sum_{k=1}^{K} m_{k}}$,

2) $\mathbb{P}\left(\left|a_{\mathrm{Sb}}\right|^{2} \leq \rho^{-v}\right) \dot{\leq} \rho^{-v \sum_{k=1}^{K} m_{k}}$,

$$
\mathbb{P}\left(\left|a_{\mathrm{bD}}\right|^{2} \leq \rho^{-v}\right) \leq \rho^{-v \sum_{k=1}^{K} m_{k}},
$$

where $m_{k} \triangleq \min \left\{m_{\mathrm{S} k}, m_{k \mathrm{D}}\right\}$ and operators $\doteq$ and $\dot{\leq}$ follow Definition 2.

Proof: We first provide the proof for policy I.

We observe that if $a_{i j}$ is a Nakagami-m r.v., then $\left|a_{i j}\right|^{2}$ is distributed according to a gamma distribution, with shape $m>0$ and scale $\theta>0$ :

$$
\mathbb{P}\left(\left|a_{i j}\right|^{2} \leq x\right)=\frac{\gamma(m, x / \theta)}{\Gamma(m)}
$$

where $\Gamma(m), \gamma(m, x)$ are the complete and lower incomplete gamma functions, respectively:

$$
\Gamma(m) \triangleq \int_{0}^{+\infty} t^{m-1} e^{-t} d t, \quad \gamma(m, x) \triangleq \int_{0}^{x} t^{m-1} e^{-t} d t .
$$

Therefore, for any $v>0$ we obtain:

$\mathbb{P}\left(\min \left\{\left|a_{\mathrm{S} k}\right|^{2},\left|a_{k \mathrm{D}}\right|^{2}\right\} \leq \rho^{-v}\right)=$

$=1-\mathbb{P}\left(\left|a_{\mathrm{S} k}\right|^{2}>\rho^{-v}\right) \mathbb{P}\left(\left|a_{k \mathrm{D}}\right|^{2}>\rho^{-v}\right)$

$=\frac{\gamma\left(m_{\mathrm{S} k}, \rho^{-v} / \theta_{\mathrm{S} k}\right)}{\Gamma\left(m_{\mathrm{S} k}\right)}+$

$+\frac{\gamma\left(m_{k \mathrm{D}}, \rho^{-v} / \theta_{k \mathrm{D}}\right)}{\Gamma\left(m_{k \mathrm{D}}\right)}-\frac{\gamma\left(m_{\mathrm{S} k}, \rho^{-v} / \theta_{\mathrm{S} k}\right)}{\Gamma\left(m_{\mathrm{S} k}\right)} \frac{\gamma\left(m_{k \mathrm{D}}, \rho^{-v} / \theta_{k \mathrm{D}}\right)}{\Gamma\left(m_{k \mathrm{D}}\right)}$.

We remark that according to [20],

$$
\gamma(m, x)=e^{-x} x^{m} \sum_{n=0}^{+\infty} \frac{\Gamma(m)}{\Gamma(m+1+n)} x^{n},
$$

and thus, for any $v>0$ and $\rho \rightarrow+\infty$,

$$
\gamma\left(m, \rho^{-v}\right) \doteq \rho^{-m v} \sum_{n=0}^{+\infty} \frac{\Gamma(m)}{\Gamma(m+1+n)} \rho^{-n v} \doteq \rho^{-m v}
$$

From (28) and (30), we observe that for any $v>0$ and $\rho \rightarrow+\infty$

$$
\mathbb{P}\left(\min \left\{\left|a_{\mathrm{S} k}\right|^{2},\left|a_{k \mathrm{D}}\right|^{2}\right\} \leq \rho^{-v}\right) \leq \rho^{-m_{k} v},
$$

where $m_{k}=\min \left\{m_{\mathrm{S} k}, m_{k \mathrm{D}}\right\}$.

We can now complete the proof of the first claim:

$$
\begin{aligned}
& \mathbb{P}\left(\min \left\{\left|a_{\mathrm{Sb}}\right|^{2},\left|a_{\mathrm{bD}}\right|^{2}\right\} \leq \rho^{-v}\right)= \\
& =\prod_{k=1}^{K} \mathbb{P}\left(\min \left\{\left|a_{\mathrm{S} k}\right|^{2},\left|a_{k \mathrm{D}}\right|^{2}\right\} \leq \rho^{-v}\right) \leq \rho^{-v \sum_{k=1}^{K} m_{k}}
\end{aligned}
$$

Since $\left|a_{\mathrm{Sb}}\right|^{2}$ and $\left|a_{\mathrm{bD}}\right|^{2}$ cannot be less than $\min \left\{\left|a_{\mathrm{S} r}\right|^{2},\left|a_{r \mathrm{D}}\right|^{2}\right\}$, the second claim follows immediately from the first claim.
We now provide the proof for policy II, observing that:

$$
\begin{aligned}
& \frac{1}{2} \min \left\{\left|a_{\mathrm{S} k}\right|^{2},\left|a_{k \mathrm{D}}\right|^{2}\right\} \leq \frac{\left|a_{\mathrm{S} k}\right|^{2}\left|a_{k \mathrm{D}}\right|^{2}}{\left|a_{\mathrm{S} k}\right|^{2}+\left|a_{k \mathrm{D}}\right|^{2}} \leq \\
& \leq \frac{\left|a_{\mathrm{Sb}}\right|^{2}\left|a_{\mathrm{bD}}\right|^{2}}{\left|a_{\mathrm{Sb}}\right|^{2}+\left|a_{\mathrm{bD}}\right|^{2}} \leq \min \left\{\left|a_{\mathrm{Sb}}\right|^{2},\left|a_{\mathrm{bD}}\right|^{2}\right\}
\end{aligned}
$$

for any relay $k \in \mathcal{S}_{\text {relay. }}$. Since $\min \left\{\left|a_{\mathrm{Sb}}\right|^{2},\left|a_{\mathrm{bD}}\right|^{2}\right\} \geq$ $\frac{1}{2} \min \left\{\left|a_{\mathrm{S} k}\right|^{2},\left|a_{k \mathrm{D}}\right|^{2}\right\}, \forall k \in \mathcal{S}_{\text {relay }}$, the first claim follows directly from claim 1 , policy I proved above. Also, since $\left|a_{\mathrm{Sb}}\right|^{2}$ and $\left|a_{\mathrm{bD}}\right|^{2}$ cannot be less than $\min \left(\left|a_{\mathrm{Sb}}\right|^{2},\left|a_{\mathrm{bD}}\right|^{2}\right)$, the second claim follows immediately.

\section{REFERENCES}

[1] J. N. Laneman and G. W. Wornell, "Distributed space-time coded protocols for exploiting cooperative diversity in wireless networks," IEEE Trans. Inform. Theory, vol. 59, pp. 2415-2525, Oct. 2003.

[2] R. U. Nabar, H. Bölcskei, and F. W. Kneubühler, "Fading relay channels: Performance limits and space-time signal design," IEEE J. Select. Areas Commun., vol. 22, no. 6, pp. 1099-1109, Aug. 2004.

[3] J. N. Laneman, D. N. C. Tse, and G. W. Wornell, "Cooperative diversity in wireless networks: Efficient protocols and outage behavior," IEEE Trans. Inform. Theory, vol. 50, no. 12, pp. 3062-3080, Dec. 2004.

[4] K. Azarian, H. E. Gamal, and P. Schniter, "On the achievable diversityvs-multiplexing tradeoff in cooperative channels," IEEE Trans. Inform. Theory, vol. 51, pp. 4152-4172, Dec. 2005.

[5] S. Yang and J.-C. Belfiore, "A novel two-relay three-slot amplify-andforward cooperative scheme," in Proc. Conf. on Inform. Sci. and Sys. Princeton, NJ, 2006.

[6] — , "Towards the optimal amplify-and-forward cooperative diversity scheme," IEEE Trans. Inform. Theory, Mar. 2006, submitted.

[7] T. Kiran and B. S. Rajan, "Partially-coherent distributed space-time codes with differential encoder and decoder," IEEE J. Select. Areas Commun., vol. 25, no. 2, pp. 426-433, Feb. 2007.

[8] A. Bletsas and A. Lippman, "Implementing cooperative diversity antenna arrays with commodity hardware," IEEE Commun. Mag., vol. 44, no. 12, pp. 33-40, Dec. 2006.

[9] B. Zhao and M. C. Valenti, "Practical relay networks: a generalization of hybrid-arq," IEEE J. Select. Areas Commun. (special issue on wireless ad hoc networks), vol. 23, no. 1, pp. 7-18, Jan. 2005.

[10] M. Zorzi and R. R. Rao, "Geographic random forwarding (geraf) for ad hoc and sensor networks: energy and latency performance," IEEE Trans. Mobile Comput., vol. 2, no. 6, pp. 337-348, Oct.-Dec. 2003.

[11] A. Bletsas, A. Khisti, D. P. Reed, and A. Lippman, "A simple cooperative diversity method based on network path selection," IEEE J. Select. Areas Commun. (special issue on 4G wireless systems), vol. 24, no. 9, pp. 659-672, Mar. 2006.

[12] M. Z. Win and J. H. Winters, "Virtual branch analysis of symbol error probability for hybrid selection/maximal-ratio combining in Rayleigh fading," IEEE Trans. Commun., vol. 49, no. 11, pp. 1926-1934, Nov. 2001.

[13] A. Conti, M. Z. Win, and M. Chiani, "On the inverse symbol error probability for diversity reception," IEEE Trans. Commun., vol. 51, no. 5, pp. 753-756, May 2003.

[14] A. Bletsas, "Intelligent antenna sharing in cooperative diversity wireless networks," Ph.D. dissertation, Massachusetts Institute of Technology, Cambridge, MA, Sept. 2005.

[15] A. Bletsas, H. Shin, and M. Z. Win, "Cooperative communications with outage-optimal opportunistic relaying," IEEE Trans. Wireless Commun., vol. 6, no. 9, Sept. 2007.

[16] _ , "Outage optimality of amplify-and-forward opportunistic relaying," IEEE Commun. Lett., vol. 11, no. 3, Mar. 2007.

[17] K. Azarian, H. E. Gamal, and P. Schniter, "On the optimality of ARQDDF protocols," IEEE Trans. Inform. Theory, Jan. 2006, submitted.

[18] L. Zheng and D. N. C. Tse, "Diversity and multiplexing: a fundamental tradeoff in multiple antenna channels," IEEE Trans. Inform. Theory, vol. 49, pp. 1073-1096, May 2003.

[19] H. E. Gamal, G. Caire, and M. O. Damen, "The MIMO ARQ channel: diversity-multiplexing-delay tradeoff," IEEE Trans. Inform. Theory, vol. 52 , no. 8 , Aug. 2006.

[20] M. Abramowitz and I. A. Stegun, Handbook of Mathematical Functions wih Formulas, Graphs, and Mathematical Tables. Washington, DC: United States Department of Commerce, 1970. 